\title{
Role of Helicobacter pylori Eradication Therapy on Platelet Recovery in Chronic Immune Thrombocytopenic Purpura
}

\author{
Khan Sheema, ${ }^{1}$ Ujjan Ikramdin, ${ }^{1}$ Naz Arshi, ${ }^{2}$ Naz Farah, ${ }^{1}$ and Sheikh Imran ${ }^{1}$ \\ ${ }^{1}$ Liaquat University of Medical and Health Sciences, Jamshoro, Pakistan \\ ${ }^{2}$ National Institute of Blood Diseases and Bone Marrow Transplantation, Karachi, Pakistan \\ Correspondence should be addressed to Ujjan Ikramdin; p.ikram70@gmail.com
}

Received 1 August 2016; Accepted 8 November 2016; Published 17 January 2017

Academic Editor: Francesco Franceschi

Copyright (C) 2017 Khan Sheema et al. This is an open access article distributed under the Creative Commons Attribution License, which permits unrestricted use, distribution, and reproduction in any medium, provided the original work is properly cited.

\begin{abstract}
Background. Idiopathic thrombocytopenic purpura (ITP) is a bleeding disorder in which the immune system destroys native platelets. In this condition an autoantibody is generated against a platelet antigen. ITP affects women more often than men and is more common in children than adults. Objective. To assess the effect of Helicobacter pylori eradication therapy (HPET) on platelet count in Helicobacter pylori associated chronic immune thrombocytopenic purpura (chronic ITP) in adult. Materials and Methods. It is an interventional prospective study conducted at Liaquat University of Medical and Health Sciences, Jamshoro, from 2014 to 2015. A set of 85 patients diagnosed with chronic ITP were included in the study via convenient sampling. Patients with platelets count $<100 \times 10^{9} / \mathrm{L}$ for $>3$ months were selected. They were posed to first-line investigations which comprised complete blood count $(\mathrm{CBC})$ and peripheral blood smear examination followed by second-line tests including bone marrow examination and Helicobacter pylori stool specific antigen (HpSA-EIA). Standard H. pylori eradication therapy was offered and the patients were assessed at regular intervals for 6 months. Results. Of the 85 study patients, 32 (37.6\%) were male and 53 (62.3\%) were female. Mean ages of $H$. pylori positive and negative subjects were $43.89 \pm 7.06$ and $44.75 \pm 7.91$ years, respectively. Bone marrow examination confirmed the diagnosis and excluded other related BM disorders. H. pylori stool antigen (HpSA) was detected in 34 (40\%) patients and hence regarded as $H$. pylori positive; the rest were negative. Treatment with eradication therapy significantly improved the mean platelet counts from $48.56 \pm 21.7 \times 10^{9} / 1$ to $94.2 \pm 26.8 \times 10^{9} / 1$. Conclusion. We concluded that the anti-H. pylori eradication therapy improves blood platelet counts in chronic immune thrombocytopenia.
\end{abstract}

\section{Introduction}

Idiopathic thrombocytopenic purpura (ITP) is an autoimmune phenomenon, hence also called immune thrombocytopenic purpura (ITP). In this disease antibody coated platelets are phagocytosed by immune cells [1]. ITP may be categorized as recently diagnosed ITP, persistent ITP (312 -month duration) and chronic ITP ( $\geq 12$ months) $[2,3]$. Association between $H$. pylori and ITP has already been established. H. pylori is also associated with similar autoimmune disorders like pernicious anemia, rheumatoid arthritis, and sicca syndrome [4].

Morphologically, H. pylori is a Gram-negative spirochete which colonizes the stomach mucosa. $H$. pylori infection is implicated in the pathogenesis of gastric and duodenal ulcers. Persistent or recurrent $H$. pylori infection is a known risk factor for gastric lymphoma and adenocarcinoma [5]. H. pylori eradication therapy (HPET) has shown promising results in the management of peptic ulcers, chronic gastritis, and even regression of lymphoma of stomach [6]. Pathogenetic mechanisms involved in $H$. pylori infection include mucosa adhesion, flagella, and urease production. Cytotoxic associated gene A (CagA) and vacuolating cytotoxin A (VacA) are proven virulent factors which interact with gastric mucosa for colonization and cause infection by $H$. pylori [7]. Cell wall lipopolysaccharide and neutrophil attracting protein (HP-NAP) also participate in the virulence chain $[8,9]$. Chronic immune thrombocytopenic purpura (chronic ITP) is linked as one of the extraintestinal manifestations of H. pylori disease [10]. Chronic ITP is reported to improve by effective $H$. pylori eradication therapy (HPET) [11, 12]. The role of HPET however remains controversial as some 
studies have reported an improvement in platelet count but others have failed to demonstrate any benefit $[4,13,14]$. This study was specifically designed to evaluate effect of $H$. pylori eradication therapy (HPET) on platelet count recovery in chronic ITP patients presenting at the Liaquat University of Medical and Health Sciences (LUMHS), Jamshoro, Sindh.

\section{Materials and Methods}

This interventional and prospective study was conducted in compliance with Helsinki's declaration 2000 and with an approval by institutional ethical committee at LUMHS, Jamshoro, from 2014 to 2015. A total of 85 subjects were recruited through convenient sampling. Written informed consent was taken. The inclusion criteria comprised diagnosed cases of chronic ITP. Patients with major comorbidities, concomitant malignancies, other autoimmune phenomenon, or aplastic anemia were excluded. A comprehensive questionnaire, including detailed history and physical examination, was filled out for each patient by a clinician.

Venous blood from each patient was collected into two EDTA containing sample collection tubes, each one $3 \mathrm{ml}$ in volume. Complete blood count (CBC), including hemoglobin $(\mathrm{Hb})$ estimation and platelet count, using automated cell analyzer (Sysmex XN 1000i Tokyo, Japan) and peripheral blood smear examination, stained with Leishman's stain, were performed on all the samples.

After the collection of stool samples in clean containers, Rapid Strip HpSA (Rapid Immunochromatography) was performed for the detection of Helicobacter pylori antigens in stool.

For bone marrow examination, aspiration was carried out under local anesthesia (2\% xylocaine); smear was prepared and stained to look for the number and morphology of megakaryocytes.

2.1. Standard H. pylori Eradication Therapy. Amoxicillin 1 gram 2x daily, Clarithromycin $500 \mathrm{mg} 2 \mathrm{x}$ daily, and a proton pump inhibitor $40 \mathrm{mg} 2 \mathrm{x}$ daily were given for a duration of 2 weeks. The eradication was confirmed with HpSA stool test on the 7 th posttreatment day [16].

2.2. Follow-Up Assessment. All the study patients were followed for a period of 6 months. These were tested for CBC on weekly basis in the first month. Thereafter, weekly monitoring was continued in those on risk of bleeding (platelet count $<10,000 / \mu \mathrm{L}$ ), while the others were checked on a two-week basis. The disease response was categorized into the following three groups:

(a) Complete response (CR): platelet count $\geq 150 \times 10^{9} 1^{-1}$

(b) Partial response (PR): platelet count 50-150 $\times 10^{9} 1^{-1}$

(c) No response (NR): platelet count $<50 \times 10^{9} 1^{-1}$ or an increase of $<20 \times 10^{9} 1^{-1}$ after at least 6 months of follow-up [15].

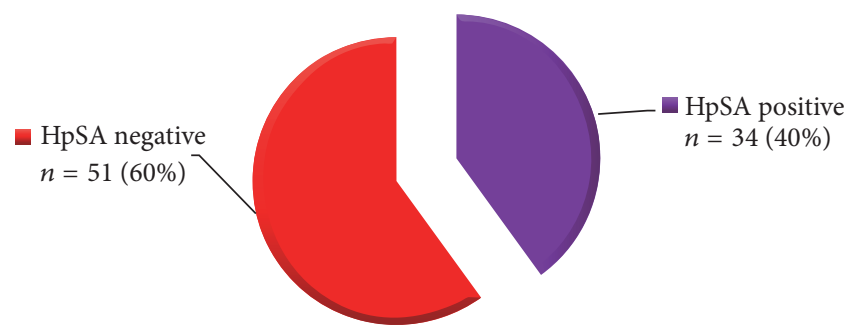

FIGURE 1: $H$. pylori infection in chronic immune thrombocytopenic purpura $(n=85)$.

2.3. Statistical Analysis. Data analysis was performed on SPSS version 22.0. Kolmogorov-Smirnov test was used for normality of data for parametric variable analysis. Continuous variables were analyzed by student's $t$-test and categorical variables by Chi-square test. $P$ level of significance was taken at $\leq 0.05$.

\section{Results}

A total of 85 patients were enrolled into the study; 32 (37.6\%) were males and $53(62.3 \%)$ were females. H. pylori positive cases were found to have a mean age of $43.89 \pm 7.06$ years, while the negative ones had a mean age of $44.75 \pm$ 7.91 years. The parameters including $\mathrm{Hb}$ and hematocrit are documented in Table 1. H. pylori stool antigen (HpSA) was detected in $34(40 \%)$ of subjects, and 51 (60\%) were HpSA negative (Figure 1). Platelet counts before $H$. pylori eradication were noted as $48.56 \pm 21.7$ million $/ \mu \mathrm{L}$ which increased to $94.2 \pm 26.8$ million $/ \mu \mathrm{L}$ after eradication therapy (Table 2). All the study patients were followed up for a period of 6 months. A significant sustained response was found in $H$. pylori eradicated cITP cases. On the other hand, $H$. pylori negative cases showed only a mild transient response. Complete recovery (CR), partial recovery (PR), and no response (NR) of platelet recovery in HpSA positive cases $(n=34)$ were observed in $19(55.8 \%), 10(29.4 \%)$, and 5 (14.7\%) cases, respectively (Table 3 ).

Bone marrow examination mostly showed increased number of megakaryocytes with abnormal morphology and distribution. Majority of the megakaryocytes were smaller in size. Other cell lineages showed normal morphology.

\section{Discussion}

An informal connection between $H$. pylori infection and immune thrombocytopenic purpura (ITP) is suggested by various clinical studies demonstrating platelet count response in approximately $50 \%$ of patients following $H$. pylori eradication [17]. Effects of $H$. pylori eradication therapy (HPET) on platelet recovery in chronic immune thrombocytopenic purpura were first reported by Gasbarrini et al. in 1998 [18]. An analysis of 25 reported series worldwide showed that eradication was successful in 671 of $792(84.7 \%)$ patients [19]. Inaba et al. had reported similar findings as $H$. pylori eradication therapy improved platelet counts [20]. The authors of present study hold the view that $H$. pylori is linked with 
TABLE 1: Baseline demographic and clinical characteristics of study patients $(N=85)$.

\begin{tabular}{lccc}
\hline Parameter & HpSA positive & HpSA negative & $P$ value \\
\hline Age (years) & $43.89 \pm 7.06$ & $44.75 \pm 7.91$ & 0.53 \\
Male & 12 & 20 & - \\
Female & 22 & 31 & - \\
Hemoglobin $(\mathrm{g} / \mathrm{dl})$ & $11.54 \pm 1.68$ & $12.19 \pm 2.06$ & 0.12 \\
Hematocrit $(\%)$ & $45.0 \pm 7.5$ & $47.0 \pm 8.0$ & 0.30 \\
RBC (million $/ \mu \mathrm{l})$ & $2.91 \pm 0.49$ & $3.34 \pm 0.55$ & 0.0001 \\
WBC $($ per $\mu \mathrm{l})$ & $6283.7 \pm 0.310$ & $6239.0 \pm 0.311$ & 0.01 \\
Platelets $\left(\times 10^{3} / \mu \mathrm{l}\right)$ & $12.3 \pm 3.7$ & $13.5 \pm 4.1$ & 0.9 \\
\hline
\end{tabular}

HpSA: Helicobacter pylori stool antigen; $N$ : total number of patients.

* Based on Student's $t$-test.

TABLE 2: Platelet counts before and after $H$. pylori eradication therapy $(N=34)$.

\begin{tabular}{llll}
\hline Platelet counts & Mean & SD & $P$ value \\
\hline Before H. pylori eradication & 48.56 & 21.75 & 0.0001 \\
After H. pylori eradication & 94.29 & 26.85 & \\
\hline
\end{tabular}

$N$ : number of patients.

TABLE 3: Evaluation of platelet counts in response to HPET in HpSA positive patients $(N=34)$.

\begin{tabular}{lccc}
\hline Platelet response & Number & Percentile (\%) & $P$ value \\
\hline Complete response $(\mathrm{Cr})^{*}$ & 19 & 55.8 \\
Partial response $(\mathrm{Pr})^{* *}$ & 10 & 29.4 & 0.0001 \\
No response $(\mathrm{Nr})^{* * *}$ & 5 & 14.7 \\
\hline
\end{tabular}

HPET: H. pylori eradication therapy; HpSA: H. pylori stool antigen.

* Platelet count $150 \times 10^{9} \mathrm{~L}^{-1}$.

${ }^{* *}$ Platelet count $50-150 \times 10^{9} \mathrm{~L}^{-1}$.

*** Platelet count $<50 \times 10^{9} \mathrm{~L}^{-1}$ or an increase of $<20 \times 10^{9} \mathrm{~L}^{-1}$ after at least 6 months of follow-up [15].

abnormal immune reaction producing platelet destruction. On the contrary, Stasi et al. and Gan et al. reported no improvement in circulating platelets after HPET [10, 21]. Geographical variations, sampling techniques and sample size, faulty study designs, $H$. pylori strains, drug compliance, drug efficacy, systemic bias, and research bias may be the main confounding factors.

The prevalence of $H$. pylori infection in chronic ITP patients varies markedly. We found $H$. pylori infection in $40 \%$ of the study cITP patients. A previous study, conducted in Italy, showed a $H$. pylori prevalence rate of $50 \%$ in cITP patients. In another study from Japan $75 \%$ of the cITP patients were found to have $H$. pylori infection. However, studies conducted in French and North American Caucasian cITP patients showed a low prevalence rate [19]. A prevalence rate of $50-80 \%$ has been marked in studies from Japan, Iran, and Korea [22-24]. It has already been shown that after HPET treatment the cITP of shorter duration responds better in comparison to the long standing ones $[1,21]$. In the current study, complete response (CR), partial response (PR), and no response (NR) of platelet recovery in HpSA positive cases $(n=$ 34 ) were noted in 19 (55.8\%), 10 (29.4\%), and 5 (14.7\%) cases, respectively. The response found was statistically significant $(P=0.0001)$. Our findings of platelet recovery are consistent with those of previous studies $[1,15,21]$. All the study patients were followed up for a period of 6 months. A significant sustained response was found in $H$. pylori eradicated cITP cases. On the other hand, $H$. pylori negative cases showed only a mild transient response. Patients with PR and NR are speculated to have other aetiologies including HIV or HCV infection or have previously been treated with interferons or other antiviral therapy interfering with megakaryopoiesis.

Brito et al. [25] and Payandeh et al. [26] reported a recovery in platelet counts after HPET treatment at 6-12month follow-up which is consistent with the current study.

\section{Conclusion}

We conclude that $40 \%$ of the local cITP patients are $H$. pylori infected and that $H$. pylori eradication therapy in these individuals significantly improves the platelet counts. Further studies on a larger cohort of patients, with a longer followup, will allow a better insight into the true prevalence of $H$. pylori infection and the duration of remission. Such studies will also conceivably endorse clarity of actual prevalence and understanding of mechanisms underlying the response to eradication therapy.

\section{Competing Interests}

The authors declare that they have no competing interests. 


\section{Authors' Contributions}

Khan Sheema designed, collected, and analyzed the data and wrote the manuscript. Ujjan Ikramdin supervised the study, helped in data analysis, and secured funding. Naz Arshi helped in manuscript writing and reviewed the data analysis. Naz Farah provided the patients samples and helped in study design. Sheikh Imran contributed to study design, specimen collection, and clinical data analysis.

\section{Acknowledgments}

The authors would like to thank all patients who participated in this study, clinicians, and staff of diagnostic and research laboratory. They also like to thank University of LUMHS for funding this project.

\section{References}

[1] R. N. Noonavath, C. P. Lakshmi, T. K. Dutta, and V. Kate, "Helicobacter pylori eradication in patients with chronic immune thrombocytopenic purpura," World Journal of Gastroenterology, vol. 20, no. 22, pp. 6918-6923, 2014.

[2] T. Kühne, W. Berchtold, L. A. Michaels et al., "Newly diagnosed immune thrombocytopenia in children and adults: a comparative prospective observational registry of the Intercontinental Cooperative Immune Thrombocytopenia Study Group," Haematologica, vol. 96, no. 12, pp. 1831-1837, 2011.

[3] D. Provan, R. Stasi, A. C. Newland et al., "International consensus report on the investigation and management of primary immune thrombocytopenia," Blood, vol. 115, no. 2, pp. 168-186, 2010.

[4] N. Rostami, M. Keshtkar-Jahromi, M. Rahnavardi, M. Keshtkar-Jahromi, and F. S. Esfahani, "Effect of eradication of Helicobacter pylori on platelet recovery in patients with chronic idiopathic thrombocytopenic purpura: a controlled trial," American Journal of Hematology, vol. 83, no. 5, pp. 376-381, 2008.

[5] S. Suerbaum and P. Michetti, "Helicobacter pylori infection," New England Journal of Medicine, vol. 347, no. 15, pp. 1175-1186, 2002.

[6] R. Sato, K. Murakami, K. Watanabe et al., "Effect of Helicobacter pylori eradication on platelet recovery in patients with chronic idiopathic thrombocytopenic purpura," Archives of Internal Medicine, vol. 164, no. 17, pp. 1904-1907, 2004.

[7] A. A. Memon, N. R. Hussein, V. Y. Miendje Deyi, A. Burette, and J. C. Atherton, "Vacuolating cytotoxin genotypes are strong markers of gastric cancer and duodenal ulcer-associated Helicobacter pylori strains: a matched case-control study," Journal of Clinical Microbiology, vol. 52, no. 8, pp. 2984-2989, 2014.

[8] L. P. Andersen, "Colonization and infection by Helicobacter pylori in humans," Helicobacter, vol. 12, no. 2, pp. 12-15, 2007.

[9] A. Amedei, A. Cappon, G. Codolo et al., "The neutrophilactivating protein of Helicobacter pylori promotes Thl immune responses," The Journal of Clinical Investigation, vol. 116, no. 4, pp. 1092-1101, 2006.

[10] G. G. Gan, A. L. Norfaizal, P. C. Bee, E. F. M. Chin, A. H. Habibah, and K. L. Goh, "Helicobacter pylori infection in chronic immune thrombocytopenic purpura patients in Malaysia," Medical Journal of Malaysia, vol. 68, no. 3, pp. 231233, 2013.
[11] H. Suzuki, F. Franceschi, T. Nishizawa, and A. Gasbarrini, "Extragastric manifestations of Helicobacter pylori infection," Helicobacter, vol. 16, no. 1, pp. 65-69, 2011.

[12] K. H. Shaikh, S. Ahmed, M. Ayyub, and J. Anwar, "Association of Helicobacter pylori infection with idiopathic thrombocytopenic purpura," Journal of the Pakistan Medical Association, vol. 59, no. 10, pp. 660-663, 2009.

[13] G. Emilia, G. Longo, M. Luppi et al., "Helicobacter pylori eradication can induce platelet recovery in idiopathic thrombocytopenic purpura," Blood, vol. 97, no. 3, pp. 812-814, 2001.

[14] E. R. Ahn, M. P. Tiede, W. Jy, C. J. Bidot, V. Fontana, and Y. S. Ahn, "Platelet activation in Helicobacter pylori-associated idiopathic thrombocytopenic purpura: eradication reduces platelet activation but seldom improves platelet counts," Acta Haematologica, vol. 116, no. 1, pp. 19-24, 2006.

[15] K. Fujimura, M. Kuwana, Y. Kurata et al., "Is eradication therapy useful as the first line of treatment in Helicobacter pyloripositive idiopathic thrombocytopenic purpura? Analysis of 207 eradicated chronic ITP cases in Japan," International Journal of Hematology, vol. 81, no. 2, pp. 162-168, 2005.

[16] D. Vaira, N. Vakil, M. Menegatti et al., "The stool antigen test for detection of Helicobacter pylori after eradication therapy," Annals of Internal Medicine, vol. 136, no. 4, pp. 280-287, 2002.

[17] D. M. Arnold, A. Bernotas, I. Nazi et al., "Platelet count response to H. pylori treatment in patients with immune thrombocytopenic purpura with and without $H$. pylori infection: a systematic review," Haematologica, vol. 94, no. 6, pp. 850-856, 2009.

[18] A. Gasbarrini, F. Franceschi, R. Tartaglione, R. Landolfi, P. Pola, and G. Gasbarrini, "Regression of autoimmune thrombocytopenia after eradication of Helicobacter pylori," The Lancet, vol. 352, no. 9131, p. 878, 1998.

[19] R. Stasi and D. Provan, "Helicobacter pylori and chronic ITP," Hematology, vol. 2008, no. 1, pp. 206-211, 2008.

[20] T. Inaba, M. Mizuno, S. Take et al., "Eradication of Helicobacter pylori increases platelet count in patients with idiopathic thrombocytopenic purpura in Japan," European Journal of Clinical Investigation, vol. 35, no. 3, pp. 214-219, 2005.

[21] R. Stasi, A. Sarpatwari, J. B. Segal et al., "Effects of eradication of Helicobacter pylori infection in patients with immune thrombocytopenic purpura: a systematic review," Blood, vol. 113, no. 6, pp. 1231-1240, 2009.

[22] M. Michel, N. Cooper, C. Jean, C. Frissora, and J. B. Bussel, "Does Helicobater pylori initiate or perpetuate immune thrombocytopenic purpura?" Blood, vol. 103, no. 3, pp. 890-896, 2004.

[23] J. Y. Yim, N. Kim, S. H. Choi et al., "Seroprevalence of Helicobacter pylori in South Korea," Helicobacter, vol. 12, no. 4, pp. 333-340, 2007.

[24] M. Michel, M. Khellaf, L. Desforges et al., "Autoimmune thrombocytopenic purpura and Helicobacter pylori infection," Archives of Internal Medicine, vol. 162, no. 9, pp. 1033-1036, 2002.

[25] H. S. H. Brito, J. A. P. Braga, S. R. Loggetto, R. S. MacHado, C. F. H. Granato, and E. Kawakami, "Helicobacter pylori infection \& immune thrombocytopenic purpura in children and adolescents: a randomized controlled trial," Platelets, vol. 26, no. 4, pp. 336-341, 2015.

[26] M. Payandeh, A. Fekri, M. Sadeghi, and E. Sadeghi, "Clinical variables among adult patients with chronic idiopathic thrombocytopenic purpura in West Iran," Iranian Journal of Blood and Cancer, vol. 7, no. 2, pp. 79-83, 2015. 


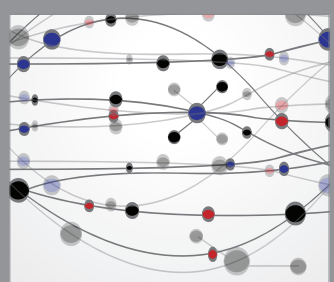

The Scientific World Journal
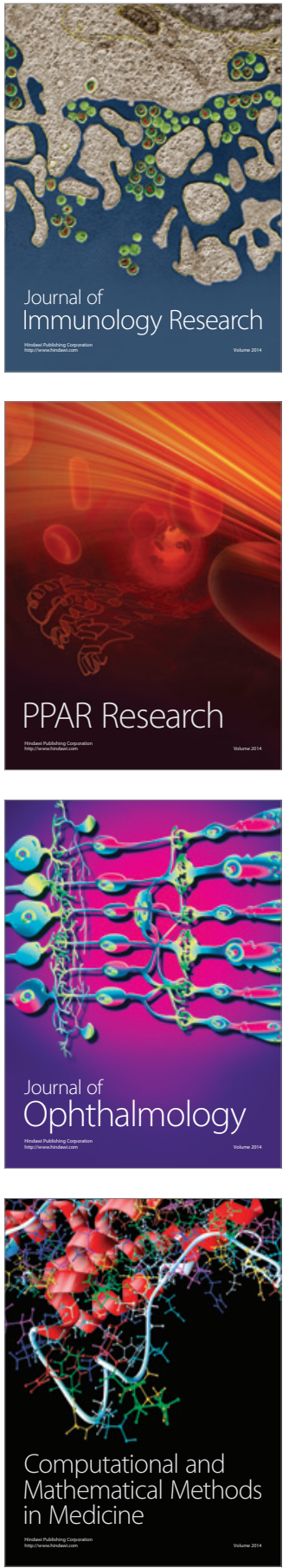

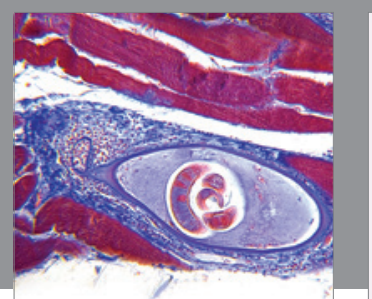

Gastroenterology Research and Practice
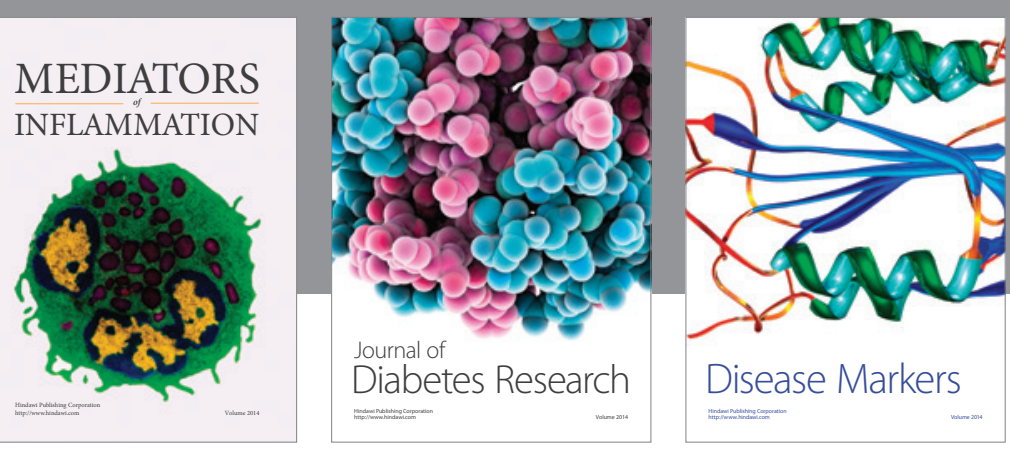

Disease Markers

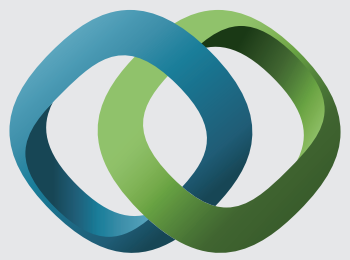

\section{Hindawi}

Submit your manuscripts at

https://www.hindawi.com
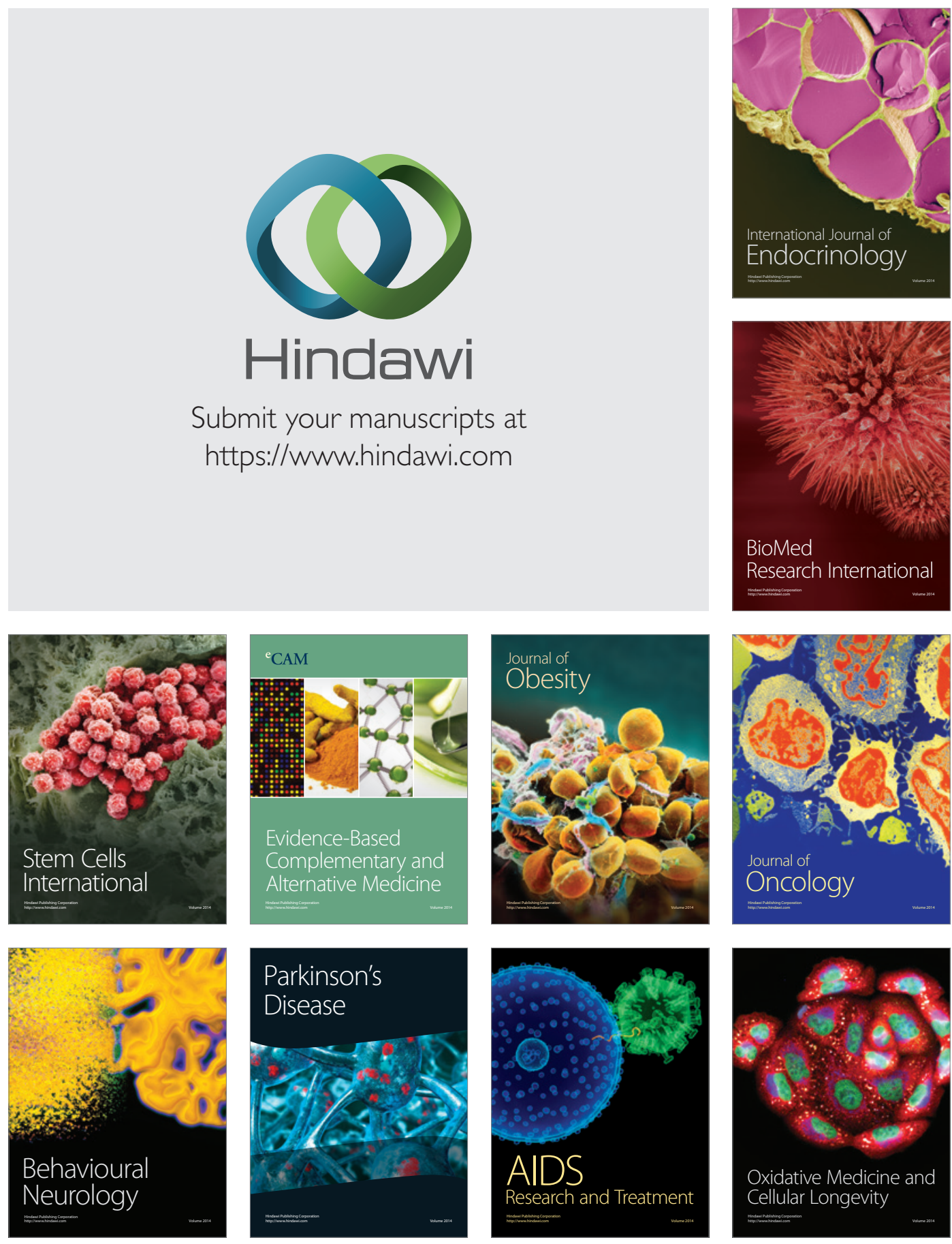